\title{
Two-Layered Susceptibility Vessel Sign and High Overestimation Ratio on MRI Are Predictive of Cardioembolic Stroke
}

\author{
(D) R. Bourcier, (DI. Derraz, ${ }^{(D)}$ S. Bracard, (D) C. Oppenheim, and (D) O. Naggara, on behalf of the THRACE Investigators
}

\begin{abstract}
SUMMARY: In a prospective study among patients in the THRombectomie des Artères Cerebrales trial, we analyzed the diagnostic accuracy of 2 imaging biomarkers, the 2-layered susceptibility vessel sign and a high overestimation ratio, obtained on pretreatment brain T2* sequences, to identify cardioembolic stroke etiology (107/260 patients). In combination, these 2 biomarkers, on 1.5T or 3T systems (159 and 101 patients, respectively), demonstrated high specificity ( 0.77 at $1.5 \mathrm{~T}$ and 1 at $3 \mathrm{~T}$ ) and their simultaneous presence is highly associated with cardioembolism.
\end{abstract}

ABBREVIATIONS: CES = cardioembolic stroke; $\mathrm{HOR}=$ high overestimation ratio; NPV = negative predictive value; overR = overestimation ratio; $\mathrm{PPV}=$ positive predictive value; SVS = susceptibility vessel sign; THRACE = THRombectomie des Artères Cerebrales; TL-SVS = 2-layered susceptibility vessel sign

$\mathbf{U}^{\prime}$ nraveling the etiology in acute ischemic stroke caused by large-vessel occlusion is important for secondary stroke prevention strategies. However, in many patients, risk factors for both large-artery atherosclerotic and cardioembolic stroke (CES) etiology are present. The composition of thrombus in obstructed arteries varies depending on whether the embolic source is cardioembolism or large-artery atherosclerosis. ${ }^{1,2}$ On admission brain MR imaging, the susceptibility vessel sign (SVS), defined as a hypointense signal exceeding the diameter of the contralateral artery at the thrombus site, ${ }^{3}$ may be seen on $\mathrm{T} 2{ }^{\star}$-weighted images. The SVS was reported to be associated with cardioembolism, but with conflicting results and low specificity. ${ }^{4-8}$ Recently, 2 characteristics of the SVS, overestimation ratio (overR), a quantitative evaluation of the SVS, and the 2-layered susceptibility vessel sign (TL-SVS), were reported to predict CES. ${ }^{7,8}$ Indeed, both the TLSVS and high overR (HOR) were described as exhibiting almost perfect specificity for CES. ${ }^{7,8}$ However, these studies were retro-

Received June 7, 2018; accepted after revision September 5.

From the Department of Neuroradiology (R.B.), University Hospital Guillaume et René Laennec, Nantes, France; Department of Neuroradiology (R.B.), University Hospital of Nancy, Nancy, France; Department of Neuroradiology and Institut $\mathrm{Na}$ tional de la Santé et de la Recherche Médicale CIC 1433 Innovation Technologique (I.D., S.B.), Université Paris-Descartes, Paris, France; Institut National de la Santé et de la Recherche Médicale U894 (C.O., O.N.), Sainte-Anne Hospital, Paris, France; and Pediatric Radiology Department (O.N.), Necker Enfants Malades, Paris, France. Catherine Oppenheim and Olivier Naggara contributed equally to this work.

Please address correspondence to Romain Bourcier, MD, PhD, Service de Neuroradiologie, Centre hospitalier universitaire Guillaume et René Laennec, Boulevard Jacques Monod, 44093 Nantes, France; e-mail: romain.bourcier2@gmail.com

Indicates article with supplemental on-line appendix.

Indicates article with supplemental on-line photo.

http://dx.doi.org/10.3174/ajnr.A5865 spective, monocentric, and used time-consuming susceptibilityweighted imaging or 3T systems, and the TL-SVS and HOR were not both evaluated in individual patients. With $>200$ patients included with a gradient-echo sequence at either $1.5 \mathrm{~T}$ or $3 \mathrm{~T}$, the multicenter THRombectomie des Artères Cerebrales (THRACE) $\operatorname{trial}^{9}$ offers an opportunity to analyze the diagnostic accuracy of these 2 imaging biomarkers for the prediction of CES.

\section{MATERIALS AND METHODS}

THRACE was a randomized controlled trial conducted in 26 centers in France. Study design and protocol are detailed elsewhere. ${ }^{10}$ Patients with acute ischemic stroke were eligible for inclusion if they were 18-80 years of age, had an NIHSS score of 10-25, had an occlusion of the intracranial ICA or the M1 segment of the MCA on MRA, could be administered intravenous thrombolysis within 4 hours of symptom onset, and could undergo thrombectomy within 5 hours of symptom onset. Patients with cervical ICA occlusion or subocclusive stenosis were excluded. Written informed consent was obtained from all patients or their legal representatives. The study protocol was approved by the Comite de Protection des Personnes III Nord Est Ethics Committee and the research boards of the participating centers.

For the present study, we included patients screened with an MR imaging gradient-echo sequence for whom analysis of the SVS was available. We excluded patients with severe motion artifacts on gradient-echo sequences when the presence or absence of SVS could not be reliably determined. We also excluded 5 patients screened with susceptibility-weighted imaging instead of a gradient-echo sequence. MR imaging acquisition parameters of the sequence were left to the discretion of the recruitment centers 


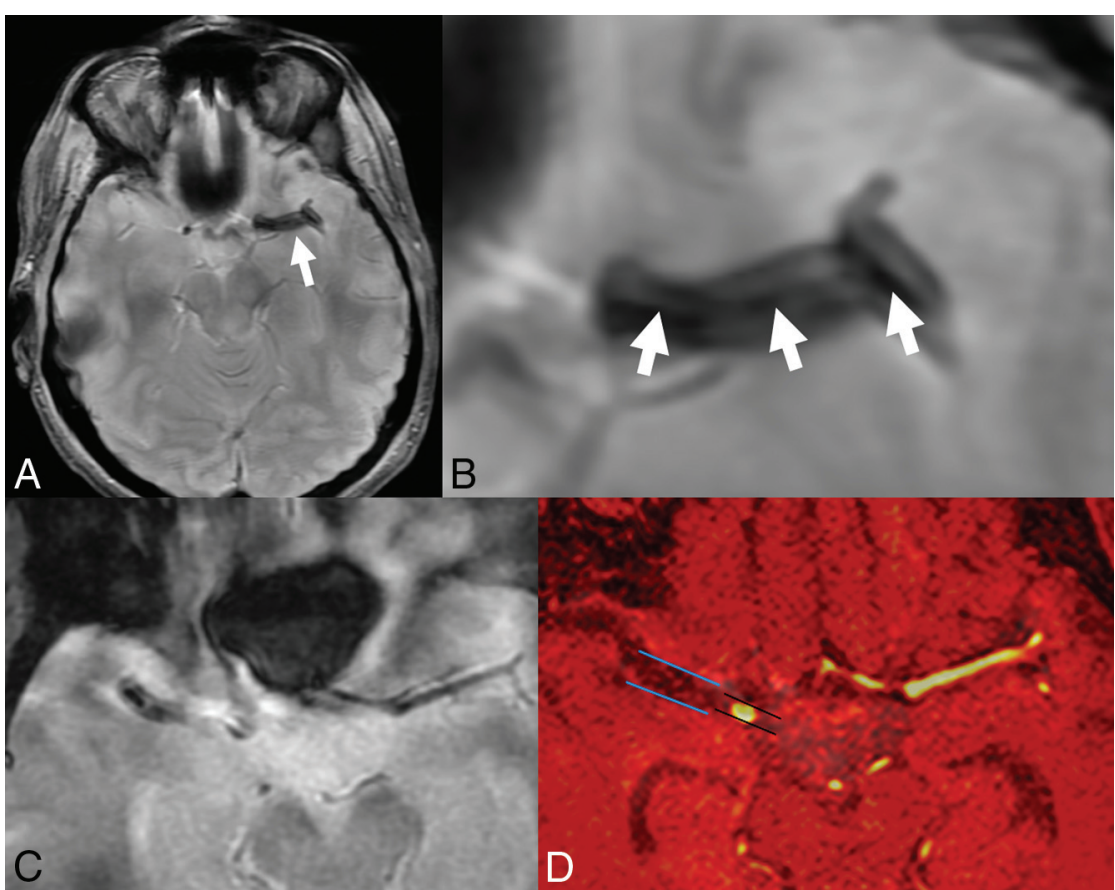

FIGURE. $A$ and $B$, Two-layered susceptibility vessel sign. A 62-year-old man with left middle cerebral artery occlusion because of cardioembolism. 1.5T T2*-weighted gradient recalled-echo imaging (white arrow) shows a 2-layered SVS with a low-intensity signal core (arrowheads) surrounded by a higher intensity signal. $C$ and $D$, Susceptibility vessel sign and overestimation ratio of SVS. A 69-year-old man with right middle cerebral artery occlusion. Overestimation ratio of SVS was 2.21 and the etiological work-up concluded to a cardiogenic embolism. The sequences of MR imaging from $C, T 2^{*}$-weighted gradient-echo images. $D$, The coregistered images of TOFMRA and $T 2^{*}$-gradient-echo.

according to their routine practice and were therefore not standardized.

Readers searched for the TL-SVS, defined as an inhomogeneous SVS with a linear low-intensity signal core surrounded by a peripheral higher intensity signal. ${ }^{8}$ The overR was manually measured according to the method previously described (ie, overR = Width of SVS / Width of Large Artery). ${ }^{7}$ The ipsilateral largeartery width proximal to the occlusion was used to calculate the overR. Note that absence of the SVS was considered as an overR $=$ 1 (Figure).

Stroke etiology was determined at the end of a complete etiologic work-up by a stroke neurologist, according to the Trial of Org 10172 in Acute Stroke Treatment classification. ${ }^{11}$ In the THRACE trial, the recommended general work-up, according to the European Stroke Organisation Guidelines, ${ }^{10}$ included physiologic parameters and routine blood tests, a 12-lead electrocardiogram, and continuous electrocardiography. In addition, 24-hour Holter electrocardiography monitoring was performed when arrhythmias were suspected and no other causes of stroke were found. The echocardiography was recommended when electrocardiographic abnormalities or evidence of cardiac disease on history was reported or when no other identifiable causes of stroke were found.

For details of the statistical analysis, see the On-line Appendix.

\section{RESULTS}

Two hundred sixty patients, including 107 (41\%) patients with CES (31/101 patients with 3T MR imaging and 76/159 patients with 1.5T MR imaging), were identified. Furthermore, the etiologic work-up found large-artery atherosclerosis in $40(15 \%)$ patients, "other determined cause of stroke" in 16 (6\%) patients, and "undetermined cause of stroke" in 97 (37\%) patients.

Overall, the median overR was 1.59 (range, 1.0-2.9; interquartile range. $0.29)$. The median overR was 1.54 (range, 1.0-2.9; interquartile range, 0.36 ) and 1.60 (range, 1.0-2.7; interquartile range, 0.54$)$ at $3 \mathrm{~T}$ and $1.5 \mathrm{~T}$, respectively.

The TL-SVS was present in $94(36 \%)$ patients (overall 43/101 patients screened at $3 \mathrm{~T}$ and $43 / 80$ patients with the SVS screened at 3T; overall 51/159 patients screened at $1.5 \mathrm{~T}$ and $51 / 136$ patients with the SVS screened at 1.5T).

At $1.5 \mathrm{~T}$, the association of the TLSVS and HOR yielded a specificity of 0.77 and a positive predictive value of 0.60 .

At $3 \mathrm{~T}$, the association of the TL-SVS and HOR yielded a specificity of 1 , a negative predictive value of 0.74 , and a positive predictive value of 1 (Tables $1-3)$.

\section{DISCUSSION}

In the present study, using $3 \mathrm{~T}$ systems, the simultaneous presence of the TL-SVS and HOR offered perfect specificity and a positive predictive value for CES. Using $1.5 \mathrm{~T}$ systems, we also showed that the simultaneous presence of the TL-SVS and HOR had 77\% specificity for CES.

Because exact stroke etiology cannot be determined with a high degree of reliability in $>30 \%$ of strokes, ${ }^{12}$ a direct relationship between imaging characteristics and stroke etiology would be useful. We demonstrated that in real world conditions (ie, brain MR imaging performed in 26 different centers on either a 1.5 or a 3T MR imaging unit, using a gradient-echo sequence of $<1$ minute), the TL-SVS and HOR can have practical implications for determining stroke etiology at the acute phase.

Recently, from the THRACE trial, qualitative visual binary grading of the SVS offered a high sensitivity of the SVS to predict a stroke of cardioembolic origin $(0.89 ; 95 \%$ CI, $0.83-0.95)$, but specificity was very low $(0.21 ; 95 \% \mathrm{CI}, 0.14-0.27) .{ }^{13}$ In the present study, we demonstrate that adding a more precise analysis of SVS characteristics (ie, TL-SVS and overR), increased specificity. This was achieved at the expense of lowering the sensitivity, as usually occurs when using a combination of biomarkers rather than a single sign. However, the combined analysis of the TL-SVS and HOR with cutoff values specific to the MR imaging system field strength increased specificity values.

Two recent retrospective studies using 3T systems analyzed separately the diagnostic accuracy of the TL-SVS and HOR for 
Table 1: Diagnostic performance of the high overestimation ratio for the prediction of a cardioembolic etiology of stroke

\begin{tabular}{llll}
\hline $\begin{array}{l}\text { 3T }(\boldsymbol{n}=101) \\
\text { overR }>2.25\end{array}$ & & $\begin{array}{c}\text { 1.5T }(\boldsymbol{n}=159) \\
\text { overR }>1.33\end{array}$ & \\
\hline Sensitivity & 0.10 & Sensitivity & 0.86 \\
Specificity & 0.94 & Specificity & 0.37 \\
PPV & 0.43 & PPV & 0.56 \\
NPV & 0.70 & NPV & 0.74 \\
Accuracy & 0.68 & Accuracy & 0.60 \\
\hline
\end{tabular}

Table 2: Diagnostic performance of the 2-layered susceptibility vessel sign for the prediction of a cardioembolic etiology of stroke

\begin{tabular}{llll}
\hline $\begin{array}{c}\text { 3T }(\boldsymbol{n}=101) \\
\text { TL-SVS }\end{array}$ & & $\begin{array}{c}\text { 1.5T }(\boldsymbol{n}=159) \\
\text { TL-SVS }\end{array}$ & \\
\hline Sensitivity & 0.45 & Sensitivity & 0.39 \\
Specificity & 0.59 & Specificity & 0.75 \\
PPV & 0.33 & PPV & 0.59 \\
NPV & 0.71 & NPV & 0.57 \\
Accuracy & 0.54 & Accuracy & 0.58 \\
\hline
\end{tabular}

Table 3: Diagnostic performance of the combined 2-layered susceptibility vessel sign and the high overestimation ratio for the prediction of a cardioembolic etiology of stroke

\begin{tabular}{llll}
\hline $\begin{array}{c}3 T(n=101) \\
\text { overR }>2.25 \text { and } \\
\text { TL-SVS }\end{array}$ & & $\begin{array}{c}1.5 T(n=159) \\
\text { overR }>1.33 \\
\text { and TL-SVS }\end{array}$ & \\
\hline Sensitivity & 0.23 & Sensitivity & 0.38 \\
Specificity & 1.00 & Specificity & 0.77 \\
PPV & 1.00 & PPV & 0.60 \\
NPV & 0.74 & NPV & 0.58 \\
Accuracy & 0.76 & Accuracy & 0.58 \\
\hline
\end{tabular}

CES prediction. The authors found specificities of $97 \%{ }^{8}$ and $91 \%,{ }^{7}$ respectively, for the TL-SVS and overR of $>2.01$ to predict a CES. ${ }^{7}$ In our study, by means of a gradient-echo sequence rather than susceptibility-weighted imaging, the specificity of the TLSVS and HOR both appeared lower on 1.5T and 3T systems.

The present prospective study is the largest to date that has evaluated the TL-SVS and overR in the context of stroke etiology. Our study has several limitations. First, THRACE was not a trial designed to specifically investigate stroke etiology. However, work-ups were performed in referral comprehensive stroke centers that ensure high quality and extensive etiologic work-ups in accordance with the current guidelines. ${ }^{10}$ Second, no information was registered on the anticoagulation regimen, the use of antiplatelet agents, or the platelet count before imaging, all factors that may have influenced the SVS. Third, the diagnostic accuracy of the SVS to predict thrombus composition varies according the MR imaging machines, ${ }^{14}$ and there was no standardization of gradient-echo sequence parameters among the 26 participating centers in the THRACE trial. In our opinion, this limitation enhances the generalizability of our findings.

\section{CONCLUSIONS}

The presence of both the TL-SVS and HOR offers a high specificity for CES etiology on both 1.5 and $3 \mathrm{~T}$ systems. When a patient with stroke has both of these imaging biomarkers, a cardioembolic source is highly likely.

\section{ACKNOWLEDGMENTS}

We thank Marc Soudant from the Institut National de la Santé CIC 1433 Epidémiologie Clinique for conducting the statistical analysis and Nick Barton for language editing.

\section{REFERENCES}

1. Marder VJ, Chute DJ, Starkman S, et al. Analysis of thrombi retrieved from cerebral arteries of patients with acute ischemic stroke. Stroke 2006;37:2086-93 CrossRef Medline

2. Boeckh-Behrens T, Kleine JF, Zimmer C, et al. Thrombus histology suggests cardioembolic cause in cryptogenic stroke. Stroke 2016;47: 1864-71 CrossRef Medline

3. Rovira A, Orellana P, Alvarez-Sabín J, et al. Hyperacute ischemic stroke: middle cerebral artery susceptibility sign at echo-planar gradient-echo MR imaging. Radiology 2004;232:466-73 CrossRef Medline

4. Brinjikji W, Duffy S, Burrows A, et al. Correlation of imaging and histopathology of thrombi in acute ischemic stroke with etiology and outcome: a systematic review. J Neurointerv Surg 2017;9:529-34 CrossRef Medline

5. Cho KH, Kim JS, Kwon SU, et al. Significance of susceptibility vessel sign on T2*-weighted gradient echo imaging for identification of stroke subtypes. Stroke 2005;36:2379-83 CrossRef Medline

6. Kang DW, Jeong HG, Kim DY, et al. Prediction of stroke subtype and recanalization using susceptibility vessel sign on susceptibilityweighted magnetic resonance imaging. Stroke 2017;48:1554-59 CrossRef Medline

7. Zhang R, Zhou Y, Liu C, et al. Overestimation of susceptibility vessel sign: a predictive marker of stroke cause. Stroke 2017;48:1993-96 CrossRef Medline

8. Yamamoto N, Satomi J, Tada Y, et al. Two-layered susceptibility vessel sign on 3 -Tesla $\mathrm{T} 2{ }^{*}$-weighted imaging is a predictive biomarker of stroke subtype. Stroke 2015;46:269-71 CrossRef Medline

9. Bracard S, Ducrocq X, Mas JL, et al; THRACE investigators. Mechanical thrombectomy after intravenous alteplase versus alteplase alone after stroke (THRACE): a randomised controlled trial. Lancet Neurol 2016;15:1138-47 CrossRef Medline

10. European Stroke Organisation (ESO) Executive Committee, ESO Writing Committee. Guidelines for management of ischaemic stroke and transient ischaemic attack 2008. Cerebrovasc Dis 2008;25: 457-507 CrossRef Medline

11. Adams HP Jr, Bendixen BH, Kappelle LJ, et al. Classification of subtype of acute ischemic stroke: definitions for use in a multicenter clinical trial-TOAST. Trial of Org 10172 in Acute Stroke Treatment. Stroke 1993;24:35-41 CrossRef Medline

12. Goldstein LB, Jones MR, Matchar DB, et al. Improving the reliability of stroke subgroup classification using the Trial of ORG 10172 in Acute Stroke Treatment (TOAST) criteria. Stroke 2001;32:1091-98 CrossRef Medline

13. Bourcier R, Derraz I, Delasalle B, et al; THRACE investigators. Susceptibility vessel sign and cardioembolic etiology in the THRACE trial. Clin Neuroradiol 2018 Jun 12. [Epub ahead of print] CrossRef

14. Bourcier R, Détraz L, Serfaty JM, et al. MRI interscanner agreement of the association between the susceptibility vessel sign and histologic composition of thrombi. J Neuroimaging 2017;27:577-82 CrossRef Medline 